\title{
A GENERALIZATION OF THE ALLEN-CAHN EQUATION
}

\author{
ALAIN MIRANVILLE ${ }^{1}$, RAMON QUINTANILLA $^{2}$
}

\begin{abstract}
Our aim in this paper is to study generalizations of the Allen-Cahn equation based on a modification of the Ginzburg-Landau free energy proposed in [25]. In particular, the free energy contains an additional term called Willmore regularization. We prove the existence, uniqueness and regularity of solutions, as well as the existence of the global attractor. Furthermore, we study the convergence to the Allen-Cahn equation, when the Willmore regularization goes to zero. We finally study the spatial behavior of solutions in a semi-infinite cylinder, assuming that such solutions exist.
\end{abstract}

\section{INTRODUCTION}

The Allen-Cahn equation,

$$
\frac{\partial u}{\partial t}-\Delta u+f(u)=0
$$

describes important processes related to phase separation in binary alloys, namely, the ordering of atoms in a lattice (see [1]). Here, $u$ is the order parameter and, typically, $f(s)=s^{3}-s$. This equation is obtained by considering the Ginzburg-Landau free energy,

$$
\Psi_{\mathrm{GL}}=\int_{\Omega}\left(\frac{1}{2}|\nabla u|^{2}+F(u)\right) d x,
$$

where $F$ is a double-well potential such that $F^{\prime}=f$ and $\Omega$ is the domain occupied by the material. Assuming a relaxation dynamics, i.e., writing

$$
\frac{\partial u}{\partial t}=-\frac{\delta \Psi_{\mathrm{GL}}}{\delta u}
$$

where $\frac{\delta}{\delta u}$ denotes a variational derivative, we obtain (1.1).

In [25] (see also [3]), the authors introduced the following modification of the GinzburgLandau free energy:

$$
\Psi_{\mathrm{AMGL}}=\int_{\Omega}\left(\gamma(\nu)\left(\frac{1}{2}|\nabla u|^{2}+F(u)\right)+\frac{\beta}{2} \omega^{2}\right) d x, \beta>0,
$$

where $\nu=\frac{\nabla u}{|\nabla u|}$ and

2010 Mathematics Subject Classification. 35B41, 35B45, 35K55.

Key words and phrases. Allen-Cahn equation, Willmore regularization, well-posedness, dissipativity, global attractor, spatial behavior. 


$$
\omega=f(u)-\Delta u
$$

Here, $\gamma(\nu)$ accounts for anisotropy effects (we also refer the reader to, e.g., [4] for a different approach to account for anisotropy effects in phase-field models), $G(u)=\omega^{2}$ is called nonlinear Willmore regularization and $\beta$ is a small regularization parameter. Such a regularization is relevant, e.g., in determining the equilibrium shape of a crystal in its own liquid matrix, when anisotropy effects are strong. Indeed, in that case, the equilibrium interface may not be a smooth curve, but may present facets and corners with slope discontinuities (see, e.g., [23]). In particular, the corresponding Cahn-Hilliard equation,

$$
\frac{\partial u}{\partial t}=\Delta \frac{\delta \Psi_{\mathrm{AMGL}}}{\delta u}
$$

is an ill-posed problem and requires regularization.

In [3], the authors proposed efficient energy stable schemes for the Cahn-Hilliard equation based on (1.4) (see also [26]); actually, in [3], the authors also considered a second regularization, based on the bi-Laplacian (i.e., $G(u)=|\Delta u|^{2}$ ) and, in that case, studied the isotropic case $\gamma(\nu) \equiv 1$ as well. We also mention that, in [14], the Cahn-Hilliard equation based on the Willmore regularization is studied in the isotropic case. There, well-posedness results and the existence of finite-dimensional attractors are obtained.

Assuming isotropy, (1.4) reads

$$
\Psi_{\mathrm{MGL}}=\int_{\Omega}\left(\frac{1}{2}|\nabla u|^{2}+F(u)+\frac{\beta}{2} \omega^{2}\right) d x
$$

and, assuming again a relaxation dynamics (i.e., (1.3)), we obtain the following generalized Allen-Cahn system:

$$
\begin{gathered}
\frac{\partial u}{\partial t}-\Delta u+f(u)+\beta \omega f^{\prime}(u)-\beta \Delta \omega=0, \\
\omega=f(u)-\Delta u .
\end{gathered}
$$

Indeed, we can write, formally,

$$
\begin{gathered}
\delta \Psi_{\mathrm{MGL}}=\int_{\Omega}(\nabla u \cdot \nabla \delta u+f(u) \delta u+\beta \omega \delta \omega) d x \\
=\int_{\Omega}\left(\nabla u \cdot \nabla \delta u+f(u) \delta u+\beta \omega f^{\prime}(u) \delta u-\beta \omega \Delta \delta u\right) d x \\
=\int_{\Omega}\left(-\Delta u+f(u)+\beta \omega f^{\prime}(u)-\beta \Delta \omega\right) \delta u d x,
\end{gathered}
$$

assuming proper boundary conditions.

The study of the isotropic case can be seen as a very first step in view of the mathematical analysis of more involved models. 
Our aim in this paper is first to prove the existence and uniqueness of solutions to (1.7)(1.8) (we also mention related (conserved) models which are studied in [5] and [6]; there the well-posedness is proved by different techniques, based on time discrete approximations and a variational minimization principle). We also prove the existence of the global attractor $\mathcal{A}$. We recall that the global attractor $\mathcal{A}$ is the smallest (for the inclusion) compact set of the phase space which is invariant by the flow (i.e., $S(t) \mathcal{A}=\mathcal{A}, \forall t \geq 0$, where $S(t)$ denotes the solution operator mapping the initial datum onto the solution at time $t$ ) and attracts all bounded sets of initial data as time goes to infinity; it thus appears as a suitable object in view of the study of the asymptotic behavior of the system. We refer the reader to [2], [20] and [24] for more details and discussions on this.

Then, we study the convergence of solutions to (1.7)-(1.8) to those of the limit AllenCahn equation, i.e., we let $\beta$ tend to zero in (1.7).

We also consider the case $\gamma \equiv-1$ in (1.4). In that case, the free energy is called functionalized Cahn-Hilliard energy in [21]. In particular, we prove the existence and uniqueness of solutions.

Finally, we study the spatial behavior of solutions in a semi-infinite cylinder for the isotropic model. Spatial decay estimates for partial differential equations are related to the Saint-Venant's principle which is both a mathematical and a thermomechanical aspect which has deserved much attention in the last years (see [9], [12] and the references therein). Such studies describe how the influence of the perturbations on a part of the boundary is damped for the points which are far away from the perturbations. Spatial decay estimates for elliptic [7], parabolic [10], [11], hyperbolic [8] and/or combinations of such [22] have been obtained in the last years. However, as far as nonlinear equations are concerned, such a knowledge is limited (see [15], [16], [17], [18] and, in particular, [19] for the Allen-Cahn equation). Our aim in this paper is also to add a new contribution to this kind of problems. More precisely, what is usual is to consider a semi-infinite cylinder whose finite end is perturbed and see what happens when the spatial variable increases. However, we do not study the existence of solutions to this problem; in fact, this does not seem to be an easy task and will be studied elsewhere. We thus assume the existence of solutions and then only study the spatial asymptotic behavior in that case.

\section{THE ISOTROPIC MODEL}

2.1. Setting of the problem. We consider the following initial and boundary value problem, in a bounded and regular domain of $\mathbb{R}^{n}, n=1,2$ or 3 , with boundary $\Gamma$ :

$$
\begin{gathered}
\frac{\partial u}{\partial t}-\Delta u+f(u)+\omega f^{\prime}(u)-\Delta \omega=0, \\
\omega=f(u)-\Delta u, \\
u=\omega=0 \text { on } \Gamma, \\
\left.u\right|_{t=0}=u_{0}
\end{gathered}
$$


(here, we take $\beta=1$ ).

As far as the nonlinear term $f$ is concerned, we make the following assumptions:

$$
\begin{gathered}
f \text { is of class } \mathcal{C}^{4}, f(0)=0, f^{\prime} \geq-c_{0}, c_{0} \geq 0, \\
f(s) s \geq c_{1} F(s)-c_{2} \geq-c_{3}, c_{1}>0, c_{2}, c_{3} \geq 0, s \in \mathbb{R},
\end{gathered}
$$

where $F(s)=\int_{0}^{s} f(\tau) d \tau$,

$$
\begin{gathered}
s f(s) f^{\prime}(s)-f(s)^{2} \geq-c_{4}, c_{4} \geq 0, s \in \mathbb{R}, \\
s f^{\prime \prime}(s) \geq 0, s \in \mathbb{R} .
\end{gathered}
$$

We can note that (2.5)-(2.7) are satisfied by polynomials of the form $f(s)=\sum_{i=1}^{2 p+1} a_{i} s^{i}$, $a_{2 p+1}>0$, and, in particular, by the usual cubic nonlinear term $f(s)=s^{3}-s$. Assumption (2.8), which allows to obtain dissipative estimates (see below), is more restrictive; it is however reasonable as it is satisfied by the cubic nonlinear term $f(s)=s^{3}-s$.

Remark 2.1. We have considered, for simplicity, Dirichlet boundary conditions (we can also note that the Allen-Cahn equation is often endowed with such boundary conditions). However, Neumann boundary conditions,

$$
\frac{\partial u}{\partial \nu}=\frac{\partial \omega}{\partial \nu}=0 \text { on } \Gamma
$$

where $\nu$ is the unit outer normal, can also be considered. In that case, we can obtain the same results by replacing (2.6) by

$$
f(s) s \geq c_{1}\left(s^{2}+F(s)\right)-c_{2} \geq-c_{3}, c_{1}>0, c_{2}, c_{3} \geq 0, s \in \mathbb{R} .
$$

We denote by $((\cdot, \cdot))$ the usual $L^{2}$-scalar product, with associated norm $\|\cdot\|$, and we denote by $\|\cdot\|_{X}$ the norm in the Banach space $X$.

Throughout the paper, the same letter $c$ (and, sometimes, $c^{\prime}$ ) denotes constants which may vary from line to line. Similarly, the same letter $Q$ denotes monotone increasing (with respect to each argument) functions which may vary from line to line.

2.2. A priori estimates. We multiply (2.1) by $\frac{\partial u}{\partial t}$ and have, integrating over $\Omega$ and by parts,

$$
\left\|\frac{\partial u}{\partial t}\right\|^{2}+\frac{1}{2} \frac{d}{d t}\|\nabla u\|^{2}+\frac{d}{d t} \int_{\Omega} F(u) d x+\left(\left(\omega f^{\prime}(u)-\Delta \omega, \frac{\partial u}{\partial t}\right)\right)=0,
$$

which yields, noting that it follows from (2.2) that

the inequality

$$
\left(\left(\omega f^{\prime}(u)-\Delta \omega, \frac{\partial u}{\partial t}\right)\right)=\frac{1}{2} \frac{d}{d t}\|\omega\|^{2}
$$




$$
\frac{d}{d t}\left(\|\nabla u\|^{2}+2 \int_{\Omega} F(u) d x+\|\omega\|^{2}\right)+2\left\|\frac{\partial u}{\partial t}\right\|^{2}=0 .
$$

In particular, it follows from (2.9) that the energy decreases along the trajectories, as expected.

We then multiply (2.1) by $u$ and obtain, owing to (2.2),

$$
\begin{gathered}
\frac{1}{2} \frac{d}{d t}\|u\|^{2}+\|\nabla u\|^{2}+((f(u), u))+\int_{\Omega} u f(u) f^{\prime}(u) d x \\
+2\left(\left(f^{\prime}(u) \nabla u, \nabla u\right)\right)+\left(\left(u f^{\prime \prime}(u) \nabla u, \nabla u\right)\right)+\|\Delta u\|^{2}=0,
\end{gathered}
$$

which yields, owing again to $(2.2)$,

$\frac{1}{2} \frac{d}{d t}\|u\|^{2}+\|\nabla u\|^{2}+((f(u), u))+\|\omega\|^{2}+\int_{\Omega}\left(u f(u) f^{\prime}(u)-f(u)^{2}\right) d x+\left(\left(u f^{\prime \prime}(u) \nabla u, \nabla u\right)\right)=0$, hence, in view of (2.6)-(2.8),

$$
\frac{d}{d t}\|u\|^{2}+c\left(\|\nabla u\|^{2}+2 \int_{\Omega} F(u) d x+\|\omega\|^{2}\right) \leq c^{\prime}, c>0 .
$$

Summing (2.9) and (2.10), we find an inequality of the form

$$
\frac{d E_{1}}{d t}+c\left(E_{1}+\left\|\frac{\partial u}{\partial t}\right\|^{2}\right) \leq c^{\prime}, c>0
$$

where

$$
E_{1}=\|u\|_{H^{1}(\Omega)}^{2}+2 \int_{\Omega} F(u) d x+\|\omega\|^{2} .
$$

In particular, it follows from (2.11) and Gronwall's lemma that

$$
E_{1}(t) \leq E_{1}(0) e^{-c t}+c^{\prime}, c>0,
$$

hence, in view of (2.5) (which yields that $\|\omega\|^{2} \geq\|\Delta u\|^{2}+\|f(u)\|^{2}-2 c_{0}\|\nabla u\|^{2}$ ), (2.12) and classical elliptic regularity results,

$$
\|u(t)\|_{H^{2}(\Omega)} \leq Q\left(\left\|u_{0}\right\|_{H^{2}(\Omega)}\right) e^{-c t}+c^{\prime}, c>0, t \geq 0 .
$$

We now multiply (2.1) by $-\Delta u$ and have, owing to $(2.2)$,

$$
\begin{aligned}
\frac{1}{2} \frac{d}{d t}\|\nabla u\|^{2}+ & \|\Delta u\|^{2}+\left(\left(f^{\prime}(u) \nabla u, \nabla u\right)\right)-\left(\left(\omega f^{\prime}(u), \Delta u\right)\right) \\
& +((\Delta f(u), \Delta u))+\|\nabla \Delta u\|^{2}=0 .
\end{aligned}
$$

Noting that, owing to the continuous embedding $H^{2}(\Omega) \subset \mathcal{C}(\bar{\Omega})$ (here, $\left.n \leq 3\right)$ and $(2.2)$, 


$$
\left|\left(\left(f^{\prime}(u) \nabla u, \nabla u\right)\right)\right|+\left|\left(\left(\omega f^{\prime}(u), \Delta u\right)\right)\right|+|((\Delta f(u), \Delta u))| \leq Q\left(\|u\|_{H^{2}(\Omega)}\right)
$$

(indeed, it follows from (2.2) that $\|\omega\| \leq Q\left(\|u\|_{H^{2}(\Omega)}\right)$ ), we obtain

$$
\frac{d}{d t}\|\nabla u\|^{2}+c\|u\|_{H^{3}(\Omega)}^{2} \leq Q\left(\|u\|_{H^{2}(\Omega)}\right), c>0 .
$$

We then multiply (2.1) by $-\Delta \frac{\partial u}{\partial t}$ and find, owing to (2.2),

We have

$$
\begin{gathered}
\left\|\nabla \frac{\partial u}{\partial t}\right\|^{2}+\frac{1}{2} \frac{d}{d t}\|\Delta u\|^{2}+\left(\left(f^{\prime}(u) \nabla u, \nabla \frac{\partial u}{\partial t}\right)\right)+\left(\left(\nabla\left(\omega f^{\prime}(u)\right), \nabla \frac{\partial u}{\partial t}\right)\right) \\
-\left(\left(\nabla \Delta f(u), \nabla \frac{\partial u}{\partial t}\right)\right)+\frac{1}{2} \frac{d}{d t}\|\nabla \Delta u\|^{2}=0 .
\end{gathered}
$$

$$
\begin{gathered}
\left|\left(\left(f^{\prime}(u) \nabla u, \nabla \frac{\partial u}{\partial t}\right)\right)\right| \leq \frac{1}{8}\left\|\nabla \frac{\partial u}{\partial t}\right\|^{2}+Q\left(\|u\|_{H^{2}(\Omega)}\right), \\
\left|\left(\left(\nabla\left(\omega f^{\prime}(u)\right), \nabla \frac{\partial u}{\partial t}\right)\right)\right| \leq\left|\left(\left(\omega f^{\prime \prime}(u) \nabla u, \nabla \frac{\partial u}{\partial t}\right)\right)\right|+\left|\left(\left(f^{\prime}(u) \nabla \omega, \nabla \frac{\partial u}{\partial t}\right)\right)\right| \\
\leq \frac{1}{8}\left\|\nabla \frac{\partial u}{\partial t}\right\|^{2}+Q\left(\|u\|_{H^{2}(\Omega)}\right)\left(\|\nabla \Delta u\|^{2}+1\right),
\end{gathered}
$$

noting that it follows from (2.2) that

$$
\|\nabla \omega\|^{2} \leq Q\left(\|u\|_{H^{2}(\Omega)}\right)+2\|\nabla \Delta u\|^{2}
$$

and, owing to Hölder's inequality and proper Sobolev embeddings,

It thus follows from (2.17)-(2.20) that

$$
\begin{gathered}
\left|\left(\left(\nabla \Delta f(u), \nabla \frac{\partial u}{\partial t}\right)\right)\right| \leq\left|\left(\left(f^{\prime \prime}(u) \Delta u \nabla u, \nabla \frac{\partial u}{\partial t}\right)\right)\right|+\left|\left(\left(f^{\prime}(u) \nabla \Delta u, \nabla \frac{\partial u}{\partial t}\right)\right)\right| \\
+\left(\left(f^{\prime \prime \prime}(u)|\nabla u|^{2} \nabla u, \nabla \frac{\partial u}{\partial t}\right)\right)|+2|\left(\left(f^{\prime \prime}(u) \nabla \nabla u \cdot \nabla u, \nabla \frac{\partial u}{\partial t}\right)\right) \mid \\
\leq \frac{1}{4}\left\|\nabla \frac{\partial u}{\partial t}\right\|^{2}+Q\left(\|u\|_{H^{2}(\Omega)}\right)\|\nabla \Delta u\|^{2} .
\end{gathered}
$$

$$
\frac{d}{d t}\left(\|\Delta u\|^{2}+\|\nabla \Delta u\|^{2}\right)+\left\|\nabla \frac{\partial u}{\partial t}\right\|^{2} \leq Q\left(\|u\|_{H^{2}(\Omega)}\right)\left(\|\nabla \Delta u\|^{2}+1\right) .
$$

We multiply (2.1) by $\Delta^{2} u$ and obtain, owing to (2.2),

$\frac{1}{2} \frac{d}{d t}\|\Delta u\|^{2}+\|\nabla \Delta u\|^{2}+\left(\left(f(u), \Delta^{2} u\right)\right)+\left(\left(\omega f^{\prime}(u), \Delta^{2} u\right)\right)-\left(\left(\Delta f(u), \Delta^{2} u\right)\right)+\left\|\Delta^{2} u\right\|^{2}=0$, which yields, noting that 


$$
\left|\left(\left(f(u), \Delta^{2} u\right)\right)\right|+\left|\left(\left(\omega f^{\prime}(u), \Delta^{2} u\right)\right)\right|+\left|\left(\left(\Delta f(u), \Delta^{2} u\right)\right)\right| \leq \frac{1}{2}\left\|\Delta^{2} u\right\|^{2}+Q\left(\|u\|_{H^{2}(\Omega)}\right),
$$

the inequality

$$
\frac{d}{d t}\|\Delta u\|^{2}+\|u\|_{H^{4}(\Omega)}^{2} \leq Q\left(\|u\|_{H^{2}(\Omega)}\right)
$$

We finally multiply (2.1) by $\Delta^{2} \frac{\partial u}{\partial t}$ and find, owing to (2.2),

$$
\begin{gathered}
\left\|\Delta \frac{\partial u}{\partial t}\right\|^{2}+\frac{1}{2} \frac{d}{d t}\|\nabla \Delta u\|^{2}+\left(\left(\Delta f(u), \Delta \frac{\partial u}{\partial t}\right)\right)+\left(\left(\Delta\left(\omega f^{\prime}(u)\right), \Delta \frac{\partial u}{\partial t}\right)\right) \\
-\left(\left(\Delta^{2} f(u), \Delta \frac{\partial u}{\partial t}\right)\right)+\frac{1}{2} \frac{d}{d t}\left\|\Delta^{2} u\right\|^{2}=0 .
\end{gathered}
$$

We have

$$
\left|\left(\left(\Delta f(u), \Delta \frac{\partial u}{\partial t}\right)\right)\right| \leq \frac{1}{8}\left\|\Delta \frac{\partial u}{\partial t}\right\|^{2}+Q\left(\|u\|_{H^{2}(\Omega)}\right) .
$$

Furthermore,

$$
\Delta\left(\omega f^{\prime}(u)\right)=f^{\prime}(u) \Delta \omega+2 f^{\prime \prime}(u) \nabla u \cdot \nabla \omega+\omega f^{\prime \prime}(u) \Delta u+\omega f^{\prime \prime \prime}(u)|\nabla u|^{2},
$$

which yields, owing to $(2.2)$,

$$
\left|\left(\left(\Delta\left(\omega f^{\prime}(u)\right), \Delta \frac{\partial u}{\partial t}\right)\right)\right| \leq \frac{1}{8}\left\|\Delta \frac{\partial u}{\partial t}\right\|^{2}+Q\left(\|u\|_{H^{3}(\Omega)}\right)\left(\left\|\Delta^{2} u\right\|^{2}+1\right) .
$$

Finally,

$$
\begin{aligned}
\Delta^{2} f(u)= & f^{\prime}(u) \Delta^{2} u+2 f^{\prime \prime}(u) \nabla \Delta u \cdot \nabla u+f^{\prime \prime}(u)|\Delta u|^{2}+2 f^{\prime \prime}(u) \nabla \nabla u \cdot \nabla \nabla u \\
& +4 f^{\prime \prime \prime}(u) \nabla \nabla u \cdot \nabla u \cdot \nabla u+f^{\prime \prime \prime}(u)|\nabla u|^{2} \Delta u+f^{(4)}(u)|\nabla u|^{4}
\end{aligned}
$$

and, proceeding as above, we can prove that

$$
\left|\left(\left(\Delta^{2} f(u), \Delta \frac{\partial u}{\partial t}\right)\right)\right| \leq \frac{1}{4}\left\|\Delta \frac{\partial u}{\partial t}\right\|^{2}+Q\left(\|u\|_{H^{3}(\Omega)}\right)\left(\left\|\Delta^{2} u\right\|^{2}+1\right) .
$$

It thus follows from (2.23)-(2.26) that

$$
\frac{d}{d t}\left(\|\nabla \Delta u\|^{2}+\left\|\Delta^{2} u\right\|^{2}\right)+\left\|\frac{\partial u}{\partial t}\right\|_{H^{2}(\Omega)}^{2} \leq Q\left(\|u\|_{H^{3}(\Omega)}\right)\left(\left\|\Delta^{2} u\right\|^{2}+1\right) .
$$


2.3. The dissipative semigroup. We first have the

Theorem 2.2. We assume that $u_{0} \in H^{2}(\Omega) \cap H_{0}^{1}(\Omega)$. Then, (2.1)-(2.4) possesses a unique variational solution $u$ such that $u \in L^{\infty}\left(\mathbb{R}^{+} ; H^{2}(\Omega) \cap H_{0}^{1}(\Omega)\right) \cap L^{\infty}\left(\tau,+\infty ; H^{4}(\Omega)\right)$ and $\frac{\partial u}{\partial t} \in L^{2}\left(0, T ; L^{2}(\Omega)\right) \cap L^{2}\left(\tau, T ; H^{2}(\Omega) \cap H_{0}^{1}(\Omega)\right), \forall \tau>0, \forall 0<\tau \leq T$. Furthermore, $\omega \in L^{\infty}\left(\mathbb{R}^{+} ; L^{2}(\Omega)\right) \cap L^{\infty}\left(\tau,+\infty ; H^{2}(\Omega) \cap H_{0}^{1}(\Omega)\right), \forall \tau>0$. Finally, for $t \geq \tau, \tau>0$, the equations are satisfied in a strong sense.

\section{Proof. a) Uniqueness:}

Let $u_{1}$ and $u_{2}$ be two solutions to (2.1)-(2.3) with initial data $u_{1,0}$ and $u_{2,0}$, respectively, where $\omega_{1}$ and $\omega_{2}$ are defined from (2.2). We set $u=u_{1}-u_{2}, \omega=\omega_{1}-\omega_{2}, u_{0}=u_{1,0}-u_{2,0}$ and have

$$
\begin{gathered}
\frac{\partial u}{\partial t}-\Delta u+f\left(u_{1}\right)-f\left(u_{2}\right)+\omega_{1} f^{\prime}\left(u_{1}\right)-\omega_{2} f^{\prime}\left(u_{2}\right)-\Delta \omega=0, \\
\omega=f\left(u_{1}\right)-f\left(u_{2}\right)-\Delta u \\
u=\omega=0 \text { on } \Gamma, \\
\left.u\right|_{t=0}=u_{0} .
\end{gathered}
$$

We multiply (2.28) by $\frac{\partial u}{\partial t}$ and have, owing to (2.29),

$$
\begin{gathered}
\left\|\frac{\partial u}{\partial t}\right\|^{2}+\frac{1}{2} \frac{d}{d t}\|\nabla u\|^{2}+\left(\left(f\left(u_{1}\right)-f\left(u_{2}\right), \frac{\partial u}{\partial t}\right)\right)+\left(\left(\omega_{1} f^{\prime}\left(u_{1}\right)-\omega_{2} f^{\prime}\left(u_{2}\right), \frac{\partial u}{\partial t}\right)\right) \\
-\left(\left(\Delta\left(f\left(u_{1}\right)-f\left(u_{2}\right)\right), \frac{\partial u}{\partial t}\right)\right)+\frac{1}{2} \frac{d}{d t}\|\Delta u\|^{2}=0 .
\end{gathered}
$$

Furthermore, owing to (2.14),

$$
\left|\left(\left(f\left(u_{1}\right)-f\left(u_{2}\right), \frac{\partial u}{\partial t}\right)\right)\right| \leq \frac{1}{8}\left\|\frac{\partial u}{\partial t}\right\|^{2}+Q\left(\left\|u_{1,0}\right\|_{H^{2}(\Omega)},\left\|u_{2,0}\right\|_{H^{2}(\Omega)}\right)\|u\|^{2}
$$

and

$$
\begin{aligned}
\mid\left(\left(\omega_{1} f^{\prime}\left(u_{1}\right)\right.\right. & \left.\left.-\omega_{2} f^{\prime}\left(u_{2}\right), \frac{\partial u}{\partial t}\right)\right)|\leq|\left(\left(\omega f^{\prime}\left(u_{1}\right), \frac{\partial u}{\partial t}\right)\right)|+|\left(\left(\omega_{2}\left(f^{\prime}\left(u_{1}\right)-f^{\prime}\left(u_{2}\right)\right), \frac{\partial u}{\partial t}\right)\right) \mid \\
& \leq \frac{1}{8}\left\|\frac{\partial u}{\partial t}\right\|^{2}+Q\left(\left\|u_{1,0}\right\|_{H^{2}(\Omega)},\left\|u_{2,0}\right\|_{H^{2}(\Omega)}\right)\|u\|_{H^{2}(\Omega)}^{2} .
\end{aligned}
$$

Here, we have used the fact that

$$
\|\omega\| \leq Q\left(\left\|u_{1,0}\right\|_{H^{2}(\Omega)},\left\|u_{2,0}\right\|_{H^{2}(\Omega)}\right)\|u\|_{H^{2}(\Omega)},
$$

which follows from (2.29). Finally, 


$$
\Delta\left(f\left(u_{1}\right)-f\left(u_{2}\right)\right)=f^{\prime}\left(u_{1}\right) \Delta u_{1}-f^{\prime}\left(u_{2}\right) \Delta u_{2}+f^{\prime \prime}\left(u_{1}\right)\left|\nabla u_{1}\right|^{2}-f^{\prime \prime}\left(u_{2}\right)\left|\nabla u_{2}\right|^{2},
$$

so that, owing once more to the continuous embedding $H^{2}(\Omega) \subset \mathcal{C}(\bar{\Omega})(n \leq 3)$,

$$
\begin{aligned}
& \left|\left(\left(\Delta\left(f\left(u_{1}\right)-f\left(u_{2}\right)\right), \frac{\partial u}{\partial t}\right)\right)\right| \leq\left|\left(\left(\left(f^{\prime}\left(u_{1}\right)-f^{\prime}\left(u_{2}\right)\right) \Delta u_{1}, \frac{\partial u}{\partial t}\right)\right)\right|+\left|\left(\left(f^{\prime}\left(u_{2}\right) \Delta u, \frac{\partial u}{\partial t}\right)\right)\right| \\
& +\left|\left(\left(\left(f^{\prime \prime}\left(u_{1}\right)-f^{\prime \prime}\left(u_{2}\right)\right)\left|\nabla u_{1}\right|^{2}, \frac{\partial u}{\partial t}\right)\right)\right|+\left|\left(\left(f^{\prime \prime}\left(u_{2}\right)\left(\left|\nabla u_{1}\right|^{2}-\left|\nabla u_{2}\right|^{2}\right), \frac{\partial u}{\partial t}\right)\right)\right| \\
& \leq\left\|f^{\prime}\left(u_{1}\right)-f^{\prime}\left(u_{2}\right)\right\|_{L^{\infty}(\Omega)}\left\|\Delta u_{1}\right\|\left\|\frac{\partial u}{\partial t}\right\|+\left\|f^{\prime}\left(u_{2}\right)\right\|_{L^{\infty}(\Omega)}\|\Delta u\|\left\|\frac{\partial u}{\partial t}\right\|
\end{aligned}
$$

$+\left\|f^{\prime \prime}\left(u_{1}\right)-f^{\prime \prime}\left(u_{2}\right)\right\|_{L^{\infty}(\Omega)}\left\|\nabla u_{1}\right\|_{L^{4}(\Omega)}^{2}\left\|\frac{\partial u}{\partial t}\right\|+\left\|f^{\prime \prime}\left(u_{2}\right)\right\|_{L^{\infty}(\Omega)}\left\|\nabla\left(u_{1}+u_{2}\right)\right\|_{L^{4}(\Omega)}\|\nabla u\|_{L^{4}(\Omega)}\left\|\frac{\partial u}{\partial t}\right\|$

$$
\leq \frac{1}{4}\left\|\frac{\partial u}{\partial t}\right\|^{2}+Q\left(\left\|u_{1,0}\right\|_{H^{2}(\Omega)},\left\|u_{2,0}\right\|_{H^{2}(\Omega)}\right)\|u\|_{H^{2}(\Omega)}^{2} .
$$

It finally follows from (2.32)-(2.35) that

$$
\frac{d}{d t}\left(\|\nabla u\|^{2}+\|\Delta u\|^{2}\right)+\left\|\frac{\partial u}{\partial t}\right\|^{2} \leq Q\left(\left\|u_{1,0}\right\|_{H^{2}(\Omega)},\left\|u_{2,0}\right\|_{H^{2}(\Omega)}\right)\|u\|_{H^{2}(\Omega)}^{2},
$$

which yields, owing to Gronwall's lemma,

$$
\left\|u_{1}(t)-u_{2}(t)\right\|_{H^{2}(\Omega)} \leq e^{Q\left(\left\|u_{1,0}\right\|_{H^{2}(\Omega)},\left\|u_{2,0}\right\|_{H^{2}(\Omega)}\right) t}\left\|u_{1,0}-u_{2,0}\right\|_{H^{2}(\Omega)},
$$

hence the uniqueness, as well as the continuity with respect to the initial data in the $H^{2}$-norm.

\section{b) Existence:}

The proof of existence of solutions is based on the a priori estimates derived in the previous section and, e.g., a standard Galerkin scheme.

In particular, it follows from (2.11)-(2.12) and (2.14) that we can construct a sequence of solutions $u_{m}$ to a proper approximated problem such that

$$
u_{m} \rightarrow u \text { in } L^{\infty}\left(0, T ; H^{2}(\Omega)\right) \text { weak star, } L^{2}\left(0, T ; L^{2}(\Omega)\right) \text { and a.e. }
$$

and

$$
\frac{\partial u_{m}}{\partial t} \rightarrow \frac{\partial u}{\partial t} \text { in } L^{2}\left(0, T ; L^{2}(\Omega)\right) \text { weak, }
$$

$\forall T>0$. The passage to the limit is then standard. Let us consider the most complicated term, namely, $\omega_{m} f^{\prime}\left(u_{m}\right)$. Here, it suffices to prove, having in mind a Galerkin scheme, that $\int_{0}^{T} \int_{\Omega}\left(\omega_{m} f^{\prime}\left(u_{m}\right)-\omega f^{\prime}(u)\right) \varphi d x d t$ tends to 0 , for $\varphi$ regular enough. Indeed, we have, noting that, owing to $(2.2), \omega_{m} \rightarrow \omega$ in $L^{2}\left(0, T ; L^{2}(\Omega)\right)$ weak, 


$$
\begin{gathered}
\left|\int_{0}^{T} \int_{\Omega}\left(\omega_{m} f^{\prime}\left(u_{m}\right)-\omega f^{\prime}(u)\right) \varphi d x d t\right| \leq\left|\int_{0}^{T} \int_{\Omega}\left(\omega_{m}-\omega\right) f^{\prime}(u) \varphi d x d t\right| \\
\quad+\left|\int_{0}^{T} \int_{\Omega} \omega_{m}\left(f^{\prime}\left(u_{m}\right)-f^{\prime}(u)\right) \varphi d x d t\right| \\
\leq\left|\int_{0}^{T} \int_{\Omega}\left(\omega_{m}-\omega\right) f^{\prime}(u) \varphi d x d t\right|+c\left\|u_{m}-u\right\|_{L^{2}\left(0, T ; L^{2}(\Omega)\right)},
\end{gathered}
$$

hence the result.

Now, it follows from (2.11)-(2.12) and (2.14) that $u \in L^{\infty}\left(\mathbb{R}^{+} ; H^{2}(\Omega)\right)$ and $\frac{\partial u}{\partial t} \in$ $L^{2}\left(0, T ; L^{2}(\Omega)\right), \forall T>0$. Then, we deduce from (2.16) that, for $r>0$ fixed,

$$
\int_{t}^{t+r}\|u\|_{H^{3}(\Omega)}^{2} d \tau \leq c(r), t \geq 0
$$

where, here and below, the constant $c(r)$ also depends on $\left\|u_{0}\right\|_{H^{2}(\Omega)}$. We thus deduce from (2.21), (2.38) and the uniform Gronwall lemma (see, e.g., [24]), that

$$
\|u(t)\|_{H^{3}(\Omega)} \leq c(r), t \geq r .
$$

We also note that it follows from (2.22) that

$$
\int_{t}^{t+r}\|u\|_{H^{4}(\Omega)}^{2} d \tau \leq c(r), t \geq 0 .
$$

Finally, it follows from (2.27), (2.39)-(2.40) and, again, the uniform Gronwall lemma that

$$
\|u(t)\|_{H^{4}(\Omega)} \leq c(r), t \geq 2 r .
$$

Noting that (2.41) holds, $\forall r>0$, we deduce that $u \in L^{\infty}\left(\tau,+\infty ; H^{4}(\Omega)\right), \forall \tau>0$, and it follows from (2.27) that $\frac{\partial u}{\partial t} \in L^{2}\left(\tau, T ; H^{2}(\Omega)\right), \forall 0<\tau \leq T$.

We also have the

Theorem 2.3. If we further assume that $u_{0} \in H^{3}(\Omega)$ (resp., $u_{0} \in H^{4}(\Omega)$ ), then $u \in$ $L^{\infty}\left(\mathbb{R}^{+} ; H^{3}(\Omega)\right)$ (resp., $u \in L^{\infty}\left(\mathbb{R}^{+} ; H^{4}(\Omega)\right)$ ) and $\frac{\partial u}{\partial t} \in L^{2}\left(0, T ; H_{0}^{1}(\Omega)\right.$ ) (resp., $\frac{\partial u}{\partial t} \in$ $\left.L^{2}\left(0, T ; H^{2}(\Omega)\right)\right), \forall T>0$.

Proof. Indeed, it follows from $(2.21)$ that $u \in L^{\infty}\left(0, T ; H^{3}(\Omega)\right)$ and from $(2.27)$ that $u \in$ $L^{\infty}\left(0, T ; H^{4}(\Omega)\right), \forall T>0$. This, combined with Theorem 1, gives the further regularity on $u$ and $\frac{\partial u}{\partial t}$.

It follows from Theorem 2.2 that we can define the family of solving operators

$$
S(t): \Phi \rightarrow \Phi, u_{0} \mapsto u(t)
$$


where $\Phi=H^{2}(\Omega) \cap H_{0}^{1}(\Omega)$. Furthermore, this family of solving operators forms a semigroup (i.e., $S(0)=\mathrm{Id}, S(t+s)=S(t) \circ S(s), t, s \geq 0$, Id being the identity operator) which is, owing to $(2.37)$, continuous in the $H^{2}$-norm.

It then follows from $(2.14)$ that $S(t)$ is dissipative in $\Phi$, in the sense that it possesses a bounded absorbing set $\mathcal{B}_{0} \subset \Phi$ (i.e., $\forall B \subset \Phi$ bounded, $\exists t_{0}=t_{0}(B) \geq 0$ such that $t \geq t_{0}$ implies $\left.S(t) B \subset \mathcal{B}_{0}\right)$. Actually, we can do better and we have the

Theorem 2.4. The semigroup $S(t)$ possesses a bounded absorbing set $\mathcal{B}_{1}$ which is bounded in $H^{4}(\Omega)$.

Proof. The proof goes as in that of the further regularity results in Theorem 2.2, except that we take $u_{0}$ in a bounded set $B \subset \Phi$ and $t \geq t_{0}$, where $t_{0} \geq 0$ is such that $t \geq t_{0}$ implies $S(t) B \subset \mathcal{B}_{0}, \mathcal{B}_{0}$ being the bounded absorbing set constructed above. As a consequence, the constants $c(r)$ in (2.38)-(2.41) no longer depend on $u_{0}$ (for $t \geq t_{0}$ ), but only on $\mathcal{B}_{0}$.

As a consequence of Theorem 2.4, we have the

Corollary 2.5. The semigroup $S(t)$ possesses the global attractor $\mathcal{A}$ which is compact in $H^{2}(\Omega)$ and bounded in $H^{4}(\Omega)$.

Remark 2.6. Having a bounded absorbing set in $H^{4}(\Omega)$, it is now not difficult to prove that the global attractor $\mathcal{A}$ has finite fractal dimension, e.g., by constructing an exponential attractor (see, e.g., [20]). Here, the finite-dimensionality means, roughly speaking, that, even though the initial phase space is infinite-dimensional, the reduced dynamics is, in some proper sense, finite-dimensional and can be described by a finite number of parameters.

\section{Convergence to the Allen-Cahn equation}

We now consider the initial and boundary value problem

$$
\begin{gathered}
\frac{\partial u^{(\beta)}}{\partial t}-\Delta u^{(\beta)}+f\left(u^{(\beta)}\right)+\beta \omega^{(\beta)} f^{\prime}\left(u^{(\beta)}\right)-\beta \Delta \omega^{(\beta)}=0 \\
\omega^{(\beta)}=f\left(u^{(\beta)}\right)-\Delta u^{(\beta)} \\
u^{(\beta)}=\omega^{(\beta)}=0 \text { on } \Gamma \\
\left.u^{(\beta)}\right|_{t=0}=u_{0}
\end{gathered}
$$

and we are interested in the limit $\beta=0$ (we can assume, without loss of generality, that $0 \leq \beta \leq 1$ ). The limit problem reads (we omit, for simplicity, the upperscript (0))

$$
\frac{\partial u}{\partial t}-\Delta u+f(u)=0
$$




$$
u=0 \text { on } \Gamma \text {, }
$$

which corresponds to the Allen-Cahn equation.

We assume, for simplicity, that $f$ is the usual cubic nonlinear term, i.e., $f(s)=s^{3}-s$. In particular, (2.5)-(2.6) and (2.8) hold, (2.7) can be replaced by the stronger condition

$$
s f(s) f^{\prime}(s)-f(s)^{2} \geq c f(s)^{2}-c^{\prime}, c>0, c^{\prime} \geq 0, s \in \mathbb{R}
$$

and

$$
\left|f^{\prime}(s)\right| \leq c|f(s)|^{\theta}+c^{\prime}, 0 \leq \theta<1, c, c^{\prime} \geq 0, s \in \mathbb{R}
$$

$\left(\theta=\frac{2}{3}\right.$ here $)$.

Theorem 3.1. For any $u_{0} \in H^{2}(\Omega) \cap H_{0}^{1}(\Omega)$, the solution $u^{(\beta)}$ to (3.1)-(3.4) converges to the solution $u$ to (3.5)-(3.7) in $L^{2}\left(0, T ; H^{1}(\Omega)\right)$ weakly, $\forall T>0$.

Proof. Repeating the estimates leading to (2.11)-(2.14), we obtain

$$
\frac{d E_{1}^{(\beta)}}{d t}+c\left(E_{1}^{(\beta)}+\left\|\frac{\partial u^{(\beta)}}{\partial t}\right\|^{2}+\beta\left\|f\left(u^{(\beta)}\right)\right\|^{2}\right) \leq c^{\prime}, c>0
$$

where

$$
E_{1}^{(\beta)}=\left\|u^{(\beta)}\right\|_{H^{1}(\Omega)}^{2}+2 \int_{\Omega} F\left(u^{(\beta)}\right) d x+\beta\left\|\omega^{(\beta)}\right\|^{2} .
$$

In particular, this yields that $u^{(\beta)}$ is bounded in $L^{2}\left(0, T ; H^{1}(\Omega)\right)$ and $L^{\infty}\left(0, T ; L^{4}(\Omega)\right)$ and $\beta^{\frac{1}{2}} \omega^{(\beta)}, \beta^{\frac{1}{2}} f\left(u^{(\beta)}\right)$ and $\frac{\partial u^{(\beta)}}{\partial t}$ are bounded in $L^{2}\left(0, T ; L^{2}(\Omega)\right)$, independently of $\beta, \forall T>$ 0 .

In order to pass to the limit, the only difficulties come from the terms $\beta \omega^{(\beta)} f^{\prime}\left(u^{(\beta)}\right)$ and $f\left(u^{(\beta)}\right)$.

We have, in view of (3.9),

$$
\begin{gathered}
\left|\beta \omega^{(\beta)} f^{\prime}\left(u^{(\beta)}\right)\right|=\left|\beta^{\frac{1}{2}-\frac{\theta}{2}} \beta^{\frac{1}{2}} \omega^{(\beta)} \beta^{\frac{\theta}{2}} f^{\prime}\left(u^{(\beta)}\right)\right| \leq c \beta^{\frac{1}{2}-\frac{\theta}{2}}\left|\beta^{\frac{1}{2}} \omega^{(\beta)}\right|\left(\left|\beta^{\frac{1}{2}} f\left(u^{(\beta)}\right)\right|^{\theta}+1\right) \\
\leq c \beta^{\frac{1}{2}-\frac{\theta}{2}}\left|\beta^{\frac{1}{2}} \omega^{(\beta)}\right|\left(\left|\beta^{\frac{1}{2}} f\left(u^{(\beta)}\right)\right|+1\right),
\end{gathered}
$$

where $c$ is independent of $\beta$, and we deduce that $\beta \omega^{(\beta)} f^{\prime}\left(u^{(\beta)}\right)$ converges to 0 (at least in $\left.L^{1}\left(0, T ; L^{1}(\Omega)\right)\right)$.

Furthermore, at least for a subsequence $\beta_{k}$ (note however that the limit problem has a unique solution), $u^{\left(\beta_{k}\right)}$ converges to $u$ in $L^{2}\left(0, T ; L^{2}(\Omega)\right)$ and, for the cubic nonlinear term, 


$$
\begin{gathered}
\left\|f\left(u^{\left(\beta_{k}\right)}\right)-f(u)\right\| \leq c \int_{\Omega}\left(\left|u^{\left(\beta_{k}\right)}\right|^{2}+|u|^{2}+1\right)\left|u^{\left(\beta_{k}\right)}-u\right| d x \\
\leq c\left(\left\|u^{\left(\beta_{k}\right)}\right\|_{L^{4}(\Omega)}^{2}+\|u\|_{L^{4}(\Omega)}^{2}+1\right)\left\|u^{\left(\beta_{k}\right)}-u\right\|,
\end{gathered}
$$

which yields that $f\left(u^{\left(\beta_{k}\right)}\right)$ converges to $f(u)$ in $L^{2}\left(0, T ; L^{2}(\Omega)\right)$.

Remark 3.2. Noting that $u^{(\beta)}$ is bounded in $L^{\infty}\left(0, T ; H^{1}(\Omega)\right)$ and that it follows from classical Aubin-Lions type theorems that $u^{\left(\beta_{k}\right)}$ converges to $u$ in $\mathcal{C}\left[(0, T] ; H^{1-\epsilon}(\Omega)\right), \forall \epsilon>0$, it is not difficult to extend the above result to a polynomial nonlinear term $f(s)=$ $\sum_{i=1}^{2 p+1} a_{i} s^{i}, a_{2 p+1}>0$, for $p \geq 1$ arbitrary in one and two space dimensions and $p=1$ or 2 in three space dimensions. Note that, in that case, (3.8)-(3.9) still hold. Indeed, in the most complicated case $n=3$ and $p=2$, we have, taking $\epsilon=\frac{1}{2}$,

$$
\begin{aligned}
& \left\|f\left(u^{\left(\beta_{k}\right)}\right)-f(u)\right\| \leq c \int_{\Omega}\left(\left|u^{\left(\beta_{k}\right)}\right|^{4}+|u|^{4}+1\right)\left|u^{\left(\beta_{k}\right)}-u\right| d x \\
& \quad \leq c\left(\left\|u^{\left(\beta_{k}\right)}\right\|_{L^{6}(\Omega)}^{4}+\|u\|_{L^{6}(\Omega)}^{4}+1\right)\left\|u^{\left(\beta_{k}\right)}-u\right\|_{L^{3}(\Omega)}, \\
& \leq c\left(\left\|u^{\left(\beta_{k}\right)}\right\|_{H^{1}(\Omega)}^{4}+\|u\|_{H^{1}(\Omega)}^{4}+1\right)\left\|u^{\left(\beta_{k}\right)}-u\right\|_{H^{\frac{1}{2}(\Omega)}} .
\end{aligned}
$$

Here, we have used the continuous Sobolev embeddings $H^{1}(\Omega) \subset L^{6}(\Omega)$ and $H^{\frac{1}{2}}(\Omega) \subset$ $L^{3}(\Omega)$

\section{Functionalized CAhn-Hilliard EnERgy}

In this section, we consider the following initial and boundary value problem (we now again take $\beta=1$ ):

$$
\begin{gathered}
\frac{\partial u}{\partial t}+\Delta u-f(u)+\omega f^{\prime}(u)-\Delta \omega=0, \\
\omega=f(u)-\Delta u, \\
u=\omega=0 \text { on } \Gamma, \\
\left.u\right|_{t=0}=u_{0} .
\end{gathered}
$$

As far as the nonlinear term $f$ is concerned, we assume that (2.5) and (2.7)-(2.8) hold. We further assume that

$$
|F(s)| \leq \epsilon f(s)^{2}+c_{\epsilon}, \forall \epsilon>0, s \in \mathbb{R} .
$$

Again, these assumptions are satisfied, in particular, by the usual cubic nonlinear term $f(s)=s^{3}-s$. 
Multiplying (4.1) by $u$ and proceeding as in Subsection 2.2, we have

$$
\frac{1}{2} \frac{d}{d t}\|u\|^{2}+\|\omega\|^{2} \leq|((f(u), u))|+\|\nabla u\|^{2}+c^{\prime}, c>0 .
$$

Noting that

$$
|((f(u), u))| \leq \epsilon\|f(u)\|^{2}+c_{\epsilon}\|u\|^{2}, \forall \epsilon>0,
$$

that, owing to (2.5),

$$
\|\omega\|^{2} \geq\|\Delta u\|^{2}+\|f(u)\|^{2}-2 c_{0}\|\nabla u\|^{2}
$$

and employing the interpolation inequality

$$
\|u\|_{H^{1}(\Omega)}^{2} \leq c\|u\|\|\Delta u\|,
$$

we then obtain

$$
\frac{d}{d t}\|u\|^{2}+c\left(\|u\|_{H^{2}(\Omega)}^{2}+\|f(u)\|^{2}\right) \leq c^{\prime}\left(\|u\|^{2}+1\right), c>0 .
$$

We now multiply (4.1) by $\frac{\partial u}{\partial t}$ and find

$$
\frac{d}{d t}\left(\|\omega\|^{2}-\|\nabla u\|^{2}-2 \int_{\Omega} F(u) d x\right)+2\left\|\frac{\partial u}{\partial t}\right\|^{2}=0,
$$

where, owing to (2.5), (4.5) and (4.9),

$$
\begin{gathered}
\|\omega\|^{2}-\|\nabla u\|^{2}-2 \int_{\Omega} F(u) d x \geq\|\Delta u\|^{2}+\|f(u)\|^{2}-\left(2 c_{0}+1\right)\|\nabla u\|^{2}-2 \int_{\Omega} F(u) d x \\
\geq \frac{1}{2}\left(\|\Delta u\|^{2}+\|f(u)\|^{2}\right)-c\left(\|u\|^{2}+1\right) .
\end{gathered}
$$

At this stage, we can proceed exactly as in Subsection 2.2 and have the following inequalities:

$$
\begin{gathered}
\frac{d}{d t}\|\nabla u\|^{2}+\|u\|_{H^{3}(\Omega)}^{2} \leq Q\left(\|u\|_{H^{2}(\Omega)}\right) \\
\frac{d}{d t}\left(\|\nabla \Delta u\|^{2}-\|\Delta u\|^{2}\right)+\left\|\frac{\partial u}{\partial t}\right\|_{H^{1}(\Omega)}^{2} \leq Q\left(\|u\|_{H^{2}(\Omega)}\right)\left(\|\nabla \Delta u\|^{2}+1\right), \\
\frac{d}{d t}\|\Delta u\|^{2}+\|u\|_{H^{4}(\Omega)}^{2} \leq Q\left(\|u\|_{H^{2}(\Omega)}\right)\left(\|\nabla \Delta u\|^{2}+1\right)
\end{gathered}
$$

and 


$$
\frac{d}{d t}\left(\left\|\Delta^{2} u\right\|^{2}-\|\nabla \Delta u\|^{2}\right)+\left\|\frac{\partial u}{\partial t}\right\|_{H^{2}(\Omega)}^{2} \leq Q\left(\|u\|_{H^{3}(\Omega)}\right)\left(\left\|\Delta^{2} u\right\|^{2}+1\right) .
$$

We then have the

Theorem 4.1. a) We assume that $u_{0} \in H^{2}(\Omega) \cap H_{0}^{1}(\Omega)$. Then, (4.1)-(4.4) possesses a unique solution $u$ such that $u \in L^{\infty}\left(0, T ; H^{2}(\Omega) \cap H_{0}^{1}(\Omega)\right) \cap L^{2}\left(0, T ; H^{4}(\Omega)\right)$ and $\frac{\partial u}{\partial t} \in$ $L^{2}\left(0, T ; L^{2}(\Omega)\right), \forall T>0$.

b) If we further assume that $u_{0} \in H^{3}(\Omega)$ (resp., $u_{0} \in H^{4}(\Omega)$ ), then $u \in L^{\infty}\left(0, T ; H^{3}(\Omega)\right.$ ) (resp., $u \in L^{\infty}\left(0, T ; H^{4}(\Omega)\right)$ ) and $\frac{\partial u}{\partial t} \in L^{2}\left(0, T ; H_{0}^{1}(\Omega)\right)$ (resp., $\frac{\partial u}{\partial t} \in L^{2}\left(0, T ; H^{2}(\Omega)\right)$ ), $\forall T>0$.

The proof of Theorem 4.1 is based on the above a priori estimates and is similar to those of Theorems 2.2-2.3.

Remark 4.2. If we further assume that (3.9) holds, for $\theta \neq 0$, then if follows from (4.1)(4.2) and (4.10) that $\frac{\partial u}{\partial t} \in L^{\frac{2}{1+\theta}}\left(0, T ; L^{\frac{2}{1+\theta}}(\Omega)\right)+L^{2}\left(0, T ; H^{-2}(\Omega)\right)$ and we deduce from (4.10) the existence of a solution $u$ such that $u \in L^{\infty}\left(0, T ; L^{2}(\Omega)\right) \cap L^{2}\left(0, T ; H^{2}(\Omega) \cap\right.$ $\left.H_{0}^{1}(\Omega)\right), \forall T>0$, for $u_{0} \in L^{2}(\Omega)$.

Remark 4.3. We can note that, here, we are not able to study the asymptotic behavior of the associated dynamical system. Indeed, the estimates derived in this section are not dissipative.

\section{Spatial BehaVior FOR THE isotropic MODEL}

The goal of this section is to study the spatial behavior of the equation determined by system (2.1)-(2.2), namely,

$$
\frac{\partial u}{\partial t}-\left(1+f^{\prime}(u)\right) \Delta u+f(u)\left(1+f^{\prime}(u)\right)-\Delta f(u)+\Delta^{2} u=0
$$

associated with null initial conditions and suitable boundary and asymptotic conditions. We study this equation in the semi-infinite cylinder $R=(0,+\infty) \times D$, where $D$ is a twodimensional bounded domain which is smooth enough to allow the use of the divergence theorem.

As we work with a fourth-order (in space) equation, we need to impose two conditions at every point of the boundary. Our equation usually is endowed with Neumann or Dirichlet boundary conditions, as done in the previous sections. However, for the sake of simplicity, we first consider mixed Dirichlet and Neumann boundary conditions, namely,

$$
\begin{gathered}
u=\frac{\partial u}{\partial \nu}=0 \text { on }(0,+\infty) \times \partial D \times(0, T), \\
u\left(0, x_{2}, x_{3}, t\right)=h\left(x_{2}, x_{3}, t\right), u_{, 1}\left(0, x_{2}, x_{3}, t\right)=l\left(x_{2}, x_{3}, t\right) \text { on }\{0\} \times D \times(0, T),
\end{gathered}
$$

where $u_{, i}$ denotes the derivative with respect to $x_{i}$ and $T>0$ is a given final time, together with null initial conditions,

$$
\left.u\right|_{t=0}=0 \text { in } R
$$


We note that, whenever conditions (5.2) on the lateral boundary of the cylinder hold, we obtain

$$
\nabla u=0 \text { on }(0,+\infty) \times \partial D \times(0, T) .
$$

Our aim is to obtain a spatial decay estimate of exponential type for the solutions to the problem determined by the equation and the initial and boundary conditions whenever the function $f(s)$ satisfies suitable conditions and the total energy contained in the cylinder is bounded ${ }^{1}$.

We assume that the nonlinear term satisfies

$$
f(s) s\left(1+f^{\prime}(s)\right) \geq-C s^{2}, 2 f^{\prime}(s)+s f^{\prime \prime}(s)+1 \geq C_{1}|s|^{k} s-C_{2}, s \in \mathbb{R},
$$

where $k, C$ and $C_{1}$ are positive constants and $C_{2}<\lambda_{1}(D), \lambda_{1}(D)$ being the first eigenvalue of the fixed membrane problem in the domain $D$. When $D$ is contained between two parallel lines at distance $l$, we know that $\lambda_{1}(D) \geq \pi^{2} / l^{2}$. Faber-Krahn inequality provides the estimate $\lambda_{1}(D) \geq \pi j_{0}^{2} /|D|$, where $|D|$ denotes the area of the domain $D$ and $j_{0} \sim$ $2.4048 \ldots$ is the first zero of the Bessel function of order zero. In fact, the equality holds when $D$ is a circle. Therefore our condition will be satisfied if at least one of the conditions $C_{2}<\pi^{2} / l^{2}$ or $C_{2}<\pi j_{0}^{2} /|D|$ holds.

In our calculations, the function

$$
\Phi(s)=\int_{0}^{s} f^{\prime}(s) s d s=s f(s)-\int_{0}^{s} f(s) d s
$$

will play an essential role and we further assume that it satisfies

$$
|\Phi(s)| \leq D_{1}|s|^{k+2}+D_{2} s^{2}, s \in \mathbb{R} .
$$

Again, $D_{1}$ and $D_{2}$ are two positive constants.

Remark 5.1. The examples that we have in mind for the function $f$ are polynomials of odd degree with positive leading term. However, it is clear that these polynomials do not always satisfy the second assumption in (5.6). Actually, this strongly depends on the geometry of the cross section of the cylinder. It is useful to consider a paradigmatic example to have a good idea of the applicability of our assumptions. If we consider the family of functions

$$
f(s)=A s^{2 p+1}-B s, A, B>0, p \in \mathbb{N},
$$

we have

$$
f^{\prime}(s)=(2 p+1) A s^{2 p}-B, f^{\prime \prime}(s)=2 p(2 p+1) A s^{2 p-1} .
$$

Thus, we see that

$$
\begin{gathered}
f(s) s\left(1+f^{\prime}(s)\right)=A s^{2 p+2}-B s^{2}\left((2 p+1) A s^{2 p}+(1-B)\right) \\
=s^{2}\left(A^{2}(2 p+1) s^{4 p}-(A(B-1)+A B(2 p+1)) s^{2 p}-B(B-1)\right) .
\end{gathered}
$$

\footnotetext{
${ }^{1}$ The word energy should be understood in a mathematical sense, i.e., as some functional which defines a measure.
} 
It is clear that the first condition in (5.6) is automatically satisfied. On the other hand,

$$
2 f^{\prime}(s)+s f^{\prime \prime}(s)+1=2 A(2 p+1)(p+1) s^{2 p}+1-B .
$$

Thus, the second condition in (5.6) is satisfied whenever $B<1+\lambda_{1}(D)$. it is worth noting that this condition is satisfied whenever $B<1+\pi^{2} / l^{2}$ or $B<1+\pi j_{0}^{2} /|D|$. It is also clear that (5.8) is satisfied by all elements in our family of functions.

We restrict our attention to the class of solutions which have a good spatial behavior. More precisely, we only consider the family of solutions such that the integral

$$
\int_{R} x_{1} \Gamma d v=\int_{0}^{+\infty} \int_{R(z)} \Gamma d v d z
$$

is bounded where $d v$ is the measure of he volume, for every $t \leq T$, where $R(z)=\{x \in$ $\left.R, x_{1}>z\right\}$ and

$$
\Gamma=u_{, i j} u_{, i j}+\left(1+2 f^{\prime}(u)+u f^{\prime \prime}(u)\right) u_{, i} u_{, i}+u f(u)\left(1+f^{\prime}(u)\right)+\theta u^{2}, \theta>0 .
$$

In particular, the solutions which enjoy the asymptotic conditions

$$
u(x, t), \quad u_{, i}(x, t), u_{, i j}(x, t) \rightarrow_{x_{1} \rightarrow+\infty} 0
$$

as $x_{1}^{-3}$, uniformly with respect to the other parameters, satisfy (5.13).

The following identities will be useful in what follows. Let $a$ be a nonnegative constant. We have

$$
\left(x_{1}-a\right) u_{, i j} u_{, i j}=\left(\left(x_{1}-a\right) P_{j}\right)_{, j}+\left(u_{, j 1} u\right)_{, j}-2 u_{, j 1} u_{, j}+\left(x_{1}-a\right) u_{, i i j j} u
$$

and

$$
\left(x_{1}-a\right) \phi(u) u_{, i} u_{, i}=\left(\left(x_{1}-a\right) \phi(u) u_{, i} u\right)_{, i}-\phi(u) u u_{, 1}-\left(x_{1}-a\right)\left(\phi(u) u_{, i}\right)_{, i} u,
$$

where

$$
P_{j}=u_{, i j} u_{, i}-u_{, i i j} u
$$

and $\phi$ is an arbitrary smooth function, for every solution to equation (5.1). We take, in what follows, $\phi(s)=1+f^{\prime}(s)$.

As

$$
u\left(f^{\prime}(u) u_{, i}\right)_{, i}=f^{\prime}(u) u u_{, i i}+u f^{\prime \prime}(u) u_{, i} u_{, i}
$$

and recalling the definition of the function $\Phi$, we also obtain

$$
\left(x_{1}-a\right)\left(f^{\prime}(u)+u f^{\prime \prime}(u)\right) u_{, i} u_{, i}=\left(\left(x_{1}-a\right) f^{\prime}(u) u_{, i} u\right)_{, i}-\Phi(u)_{, 1}-\left(x_{1}-a\right)\left(f^{\prime}(u) u_{, i}\right)_{, i} u .
$$

We thus see that the equality

$$
\int_{R(z)}(\xi-z)\left(\Gamma^{*}+u_{t} u\right) d v=-\int_{D(z)}\left(u_{, 11} u-u_{, j} u_{, j}-\Psi(u)\right) d a
$$

holds, for every solution to the problem satisfying (5.13), where $\Psi(s)=2 \Phi(s)+s^{2} / 2$ and

$$
\Gamma^{*}=u_{, i j} u_{, i j}+\left(1+2 f^{\prime}(u)+u f^{\prime \prime}(u)\right) u_{, i} u_{, i}+u f(u)\left(1+f^{\prime}(u)\right) .
$$

Here and below, $D(z)=\left\{x \in R, x_{1}=z\right\}$. 
The main point of our argument (for the above boundary conditions) is the choice of a suitable function $H(s) \geq 0$, for every $s \geq 0$, satisfying the differential inequality

$$
H^{\prime \prime}(s) \geq C^{2} H(s), C \in \mathbb{R}, s \geq 0 .
$$

We start our analysis by considering the functional

$$
H_{\theta}(z, t)=-\int_{0}^{t} \int_{D(z)} \exp (-2 \theta s)\left(u_{, 11} u-u_{, j} u_{, j}-\Psi(u)\right) d a d s,
$$

where $\theta$ is an arbitrary positive constant to be fixed below.

Owing to the boundary and initial conditions and to the divergence theorem, we have

$$
\frac{\partial^{2} H_{\theta}}{\partial z^{2}}(z, t)=\int_{0}^{t} \int_{D(z)} \exp (-2 \theta s) \Gamma d a d s+\exp (-2 \theta t) \int_{D(z)} \frac{u^{2}}{2} d a .
$$

The Poincaré inequality then implies

$$
\frac{\partial^{2} H_{\theta}}{\partial z^{2}}(z, t) \geq C_{3} \int_{0}^{t} \int_{D(z)} \exp (-2 \theta s)\left[u_{, i j} u_{, i j}+|u|^{k} u_{, i} u_{, i}+u_{, i} u_{, i}+u^{2}\right] d a d s,
$$

for a suitable constant $C_{3}>0$.

Performing two quadratures, we find

$$
H_{\theta}(z, t)=\int_{0}^{t} \int_{R(z)}(\xi-z) \exp (-2 \theta s) \Gamma d v d s+\exp (-2 \theta t) \int_{R(z)}(\xi-z) \frac{u^{2}}{2} d v .
$$

In view of the assumptions made and the Poincaré inequality, we see that $H_{\theta}$ is a nonnegative function satisfying the differential inequality (5.17), for a suitable positive constant $C$. It is well known that, in that case, the function $H_{\theta}$ satisfies the decay estimate (see [12])

$$
H_{\theta}(z, t) \leq H_{\theta}(0, t) \exp \left(-C^{*} z\right),
$$

for every $z \geq 0^{2}$. Therefore, we obtain the decay bound

$$
\int_{0}^{t} \int_{R(z)}(\xi-z) \Gamma d v d s \leq H_{\theta}(0, t) \exp \left(2 \theta t-C^{*} z\right) .
$$

Here, $C^{*}$ is a positive constant which can be computed explicitly. We have thus proved the

Theorem 5.2. Let $u$ be a solution to the problem determined by equation (5.1), the boundary conditions (5.2)-(5.3) and the null initial condition (5.4) which satisfies (5.13). Then, u satisfies estimate (5.23).

We now wish to describe how we can extend our argument to Dirichlet boundary conditions, namely,

$$
u=\Delta u=0 \text { on }(0,+\infty) \times \partial D \times(0, T),
$$

\footnotetext{
${ }^{2}$ In general, from (5.17), we can obtain an increase/decay alternative on the solutions, but, in view of (5.13), only the decay estimate can hold.
} 
on the lateral surface of the cylinder. We must also assume a condition of the form

$$
u\left(0, x_{2}, x_{3}, t\right)=h\left(x_{2}, x_{3}, t\right), u_{, 11}\left(0, x_{2}, x_{3}, t\right)=l\left(x_{2}, x_{3}, t\right) \text { on }\{0\} \times D \times(0, T) .
$$

From now on, we assume that the first condition in (5.6) and condition (5.8) on the function $f$ hold. However, we need to change the second assumption in (5.6) and assume instead that

$$
2 f^{\prime}(s)+s f^{\prime \prime}(s)+1 \geq C_{1}|s|^{k} s+C_{2}, s \in \mathbb{R},
$$

where $C_{1}$ and $C_{2}$ are positive constants. We can again consider polynomials with positive leading coefficient of the form mentioned in Remark 5.1. In view of the calculations made in this remark, we see that our condition is satisfied whenever $B<1$.

The following identity will be useful in what follows:

$$
\left(x_{1}-a\right) u_{, i i} u_{, j j}=\left(\left(x_{1}-a\right) Q_{j}\right)_{, j}+\left(u_{, i i} u\right)_{, 1}-2 u_{, i i} u_{, 1}+\left(x_{1}-a\right) u_{, i i j j} u,
$$

where

$$
Q_{j}=u_{, i i} u_{, j}-u_{, i i j} u
$$

We now assume that

$$
\int_{R} x_{1} \Gamma_{1} d v=\int_{0}^{+\infty} \int_{R(z)} \Gamma_{1} d v d z
$$

is bounded ${ }^{3}$, for every $t \leq T$, where

$$
\Gamma_{1}=u_{, i i} u_{, j j}+\left(1+2 f^{\prime}(u)+u f^{\prime \prime}(u)\right) u_{, i} u_{, i}+u f(u)\left(1+f^{\prime}(u)\right)+\theta u^{2}, \theta>0 .
$$

Introducing the function

$$
H_{\theta}^{*}(z, t)=\int_{0}^{t} \int_{R(z)}(\xi-z) \exp (-2 \theta s) \Gamma_{1} d v d s+\exp (-2 \theta t) \int_{R(z)}(\xi-z) \frac{u^{2}}{2} d v,
$$

we see that

$$
H_{\theta}^{*}(z, t)=-\int_{0}^{t} \int_{D(z)} \exp (-2 \theta s)\left(u_{, i i} u-\Psi(u)\right) d a d s-2 \int_{0}^{t} \int_{R(z)} u_{, i i} u_{, 1} d v d s .
$$

The function $H_{\theta}^{*}$ satisfies two inequalities similar to (5.20) for the first two spatial derivatives, namely, there exists a positive constant $C_{4}$ such that

$$
\frac{\partial H_{\theta^{*}}}{\partial z}(z, t) \leq-C_{4} \int_{0}^{t} \int_{R(z)} \exp (-2 \theta s)\left[u_{, i i} u_{, j j}+|u|^{k} u_{, i} u_{, i}+u_{, i} u_{, i}+u^{2}\right] d a d s
$$

and

$$
\frac{\partial^{2} H_{\theta}^{*}}{\partial z^{2}}(z, t) \geq C_{4} \int_{0}^{t} \int_{D(z)} \exp (-2 \theta s)\left[u_{, i i} u_{, j j}+|u|^{k} u_{, i} u_{, i}+u_{, i} u_{, i}+u^{2}\right] d a d s .
$$

Owing to (5.31) and these last two inequalities, we deduce the existence of two positive constants $K_{1}, K_{2}$ such that the inequality

$$
H_{\theta}^{*} \leq-K_{1} \frac{\partial H_{\theta}^{*}}{\partial z}+K_{2}^{-1} \frac{\partial^{2} H_{\theta}^{*}}{\partial z^{2}}
$$

\footnotetext{
${ }^{3}$ In a similar way to condition (5.13), if $u(x, t), u_{, i}(x, t)$ and $u_{, i i}(x, t)$ tend to zero as $x_{1}^{-3}$ when $x_{1}$ becomes unbounded, then condition (5.28) holds.
} 
holds (see [13]). It then follows that ${ }^{4}$

$$
H_{\theta}^{*}(z, t) \leq H_{\theta}^{*}(0, t) \exp \left(-\gamma x_{1}\right)
$$

where

$$
\gamma=\frac{1}{2}\left(\sqrt{K_{1}^{2} K_{2}^{2}+4 K_{2}}-K_{1} K_{2}\right)
$$

Therefore, we obtain the estimate

$$
\int_{0}^{t} \int_{R(z)}(\xi-z) \Gamma_{1} d v d s \leq H_{\theta}^{*}(0, t) \exp (2 \theta t-\gamma z) .
$$

We have thus proved the

Theorem 5.3. Let $u$ be a solution to the problem determined by equation (5.1), the boundary conditions (5.24)-(5.25) and the null initial condition (5.4) which satisfies (5.28). Then, u satisfies estimate (5.36).

Acknowledgments. This paper was initiated while A.M. was visiting the Universitat Politècnica de Catalunya in Terrassa. He wishes to thank this institution for its warm hospitality.

\section{REFERENCES}

[1] S.M. Allen and J.W. Cahn, A microscopic theory for antiphase boundary motion and its application to antiphase domain coarsening, Acta Metall. 27 (1979), 1085-1095.

[2] A.V. Babin and M.I. Vishik, Attractors of evolution equations, North-Holland, Amsterdam, 1992.

[3] F. Chen and J. Shen, Efficient energy stable schemes with spectral discretization in space for anisotropic Cahn-Hilliard systems, Commun. Comput. Phys. 13 (2013), 1189-1208.

[4] X. Chen, G. Caginalp and E. Esenturk, Interface conditions for a phase field model with anisotropic and non-local interactions, Arch. Ration. Mech. Anal. 202 (2011), 349-372.

[5] P. Colli and Ph. Laurençot, A phase-field approximation of the Willmore flow with volume constraint, Interfaces Free Bound. 13 (2011), 341-351.

[6] P. Colli and Ph. Laurençot, A phase-field approximation of the Willmore flow with volume and area constraints, SIAM J. Math. Anal. 44 (2012), 3734-3754.

[7] J.N. Flavin, R.J. Knops and L.E. Payne, Decay estimates for the constrained elastic cylinder of variable cross-section, Quart. Appl. Math. 47 (1989), 325-350.

[8] J.N. Flavin, R.J. Knops and L.E. Payne, Energy bounds in dynamical problems for a semi-infinite elastic beam, in "Elasticity: Mathematical Methods and Applications", G. Eason and R.W. Ogden eds., Chichester: Ellis Horwood, 101-111, 1989.

[9] C.O. Horgan, Recent developments concerning Saint-Venant's principle: A second update, Appl. Mech. Reviews 49 (1996), 101-111.

[10] C.O. Horgan, L.E. Payne and L.T. Wheeler Spatial decay estimates in transient heat conduction, Quart. Appl. Math. 42 (1984), 119-127.

[11] C.O. Horgan and R. Quintanilla, Spatial decay of transient end effects in functionally graded heat conducting materials, Quart. Appl. Math. 59 (2001), 529-542.

[12] H.A. Levine and R. Quintanilla, Some remarks on Saint-Venant's principle, Math. Methods Appl. Sci. 11 (1989), 71-77.

[13] C. Lin and L. E. Payne, Spatial decay bounds in time dependent pipe flow of an incompressible viscous fluid, SIAM J. Appl. Math. 65 (2004), 458-474.

[14] A. Miranville, Asymptotic behavior of a sixth-order Cahn-Hilliard system, submitted.

\footnotetext{
${ }^{4}$ Again, our inequality implies an increase/decay alternative, but condition (5.28) is only compatible with the decay estimate.
} 
[15] A. Miranville and R. Quintanilla, A generalization of the Caginalp phase-field system based on the Cattaneo law, Nonlinear Anal. TMA 71 (2009), 2278-2290.

[16] A. Miranville and R. Quintanilla, Some generalizations of the Caginalp phase-field system, Appl. Anal. 88 (2009), 877-894.

[17] A. Miranville and R. Quintanilla, A phase-field model based on a three-phase-lag heat conduction, Appl. Math. Optim. 63 (2011), 133-150.

[18] A. Miranville and R. Quintanilla, On a phase-field system based on the Cattaneo law, Nonlinear Anal. TMA 75 (2012), 2552-2565.

[19] A. Miranville and R. Quintanilla, Spatial decay for several phase-field models, Jour. Appl. Math. Mech. (ZAMM) DOI 10.1002/zamm.201200131 (2013), 1-10.

[20] A. Miranville and S. Zelik, Attractors for dissipative partial differential equations in bounded and unbounded domains, in Handbook of Differential Equations, Evolutionary Partial Differential Equations, Vol. 4, C.M. Dafermos and M. Pokorny eds., Elsevier, Amsterdam, 103-200, 2008.

[21] K. Promislow and H. Zhang, Critical points of functionalized Lagrangians, Discrete Cont. Dyn. System 33 (2013), 1231-1246.

[22] R. Quintanilla, Damping of end effects in a thermoelastic theory, Appl. Math. Letters 14 (2001), $137-141$.

[23] J.E. Taylor and J.W. Cahn, Diffuse interfaces with sharp corners and facets: phase-field models with strongly anisotropic surfaces, Phys. D 112 (1998), 381-411.

[24] R. Temam, Infinite-dimensional dynamical systems in mechanics and physics, Second edition, Applied Mathematical Sciences, Vol. 68, Springer-Verlag, New York, 1997.

[25] S. Torabi, J. Lowengrub, A. Voigt and S. Wise, A new phase-field model for strongly anisotropic systems, Proc. R. Soc. A 465 (2009), 1337-1359.

[26] S.M. Wise, C. Wang and J.S. Lowengrub, Solving the regularized, strongly anisotropic Cahn-Hilliard equation by an adaptative nonlinear multigrid method, J. Comput. Phys. 226 (2007), 414-446.

${ }^{1}$ Université DE Poitiers

Laboratoire de Mathématiques et Applications

UMR CNRS 7348 - SP2MI

Boulevard Marie et Pierre Curie - Téléport 2

F-86962 Chasseneuil Futuroscope Cedex, France

E-mail address: Alain.Miranville@math.univ-poitiers.fr

${ }^{2}$ ETSEIAT - UPC

Matemàtica Aplicada 2

Colom 11

S-08222 Terrassa, Barcelona, Spain

E-mail address: Ramon.Quintanilla@upc.edu 